\title{
Space and Human Consciousness: The Great Whisper
}

\author{
Abdullah A. Alabdulgader \\ Senior Congenital Cardiologist, Invasive Electrophysiologist and Cardiac Astrophysicist, Prince Sultan Cardiac \\ Center (Founder \& Chief Physician), Al-Hofuf, Saudi Arabia
}

Correspondence to: Abdullah A. Alabdulgader, kidsecho@yahoo.com

Keywords: Space, Consciousness, Whisper, Heart Rate Variability Frequencies (HRV), Heart Based Resonant Frequencies Theory (HBRF), Solar Geomagnetic Activity (S-GMA)

Received: May 18, $2021 \quad$ Accepted: July 6, $2021 \quad$ Published: July 9, 2021

Copyright $\odot 2021$ by author(s) and Scientific Research Publishing Inc.

This work is licensed under the Creative Commons Attribution International License (CC BY 4.0).

http://creativecommons.org/licenses/by/4.0/

\section{(c) (i) Open Access}

\section{ABSTRACT}

Human Consciousness is one of most elusive issues in the scientific history. Its nature created major historical debate started thousands of years ago and still ongoing. Despite the explosive developments in the last century to explore its nature, the knowledge about it is still deficient. The important advances in the twentieth and 21 st centuries in understanding cerebral cortex dynamics fortified by the dominant materialistic approach of the era dictated its impact on consciousness science, which is perceived as sole human brain function. This original review is a call for holistic perception of human consciousness incorporating the ancient wisdom of the human civilizations with the massive current era advances in different disciplines of applied sciences. The description of René Descartes in the 17th century of the Cartesian dualism is timely to revisit with new holistic perspective, in view of the major advances of our understanding of heart brain communications, astrophysical resonances with human heart ascending afferents to central nervous system, and signaling between humans and the space. Universal vibrations, frequencies and resonances as perceived by Nikola Tesla constitute the core of our new conceptual and experimental perspective on human consciousness. Neural and psychological correlates of human consciousness which dominate the consciousness research nowadays should undergo revolutionary conceptual understanding to perceive consciousness as a massive universal event expanding from human genes to galaxies. In the next discussion, we are going to navigate in the nature and fate of human consciousness with new innovative universal perspective based on heart rate variability of human heart and its cosmic resonances as represented by Schumann Resonances, Solar Wind Indices and Galactic Cosmic Rays. The interpretation of our existential secrets and biology without the space around us is a major gap in our scientific perception of life. The delicate orchestration between human heart and the space frequencies create the great whisper which in our perspective, encodes the secrets of human consciousness. 


\section{INTRODUCTION}

It is conspicuous from our publications and others that human consciousness and its basic understanding demands serious universal holistic perspective [1] [2]. Human consciousness is a unique universal event which demands the dynamic interaction of biology, quantum physics, astrobiology, geophysics philosophy and other related disciplines. Two fundamental conceptual understandings are critical to dive and mine in the science of consciousness. First, it is believed that mind and matter are spectrum of one dimension. Compelling concept which deserves full contemplation essential to preserve intellectual alignment between ecosystems is the idea that matter owns mind and certain degree of consciousness is conceivably a feature of all micro universe and marco universe creatures. David Bohm viewed, mind and matter as projections into our explicate order from the underlying implicate order. Second is the concept of resonance and its implementations as relatively new scientific understanding in physiological as well as astrophysical rhythms. It is of paramount role for what we are calling for in the direction of holistic approach for human consciousness [1] [2]. Resonance (from Latin resonāre means to resound) refers to a phenomenal where increased amplitude will result when the frequency of a periodically applied force (or a Fourier component of it) is equal or close to a natural frequency of the system on which it acts. Since the 1970s, many authors have hypothesized that the effect of variations in the geomagnetic field (GMF) on the human body is resonant. Nikola Tesla, the renowned scientist and the hero of electrical and mechanical engineering, has a famous say: "If you want to find the secrets of the universe, think in terms of energy, frequency and vibration". Oscillating system, such as a swing, will oscillate more strongly when it is exposed to a periodic force that is applied with the same frequency as that of the oscillating system. Resonance in physics can be understood as excited states of more stable particles or the condition of a system in which there is a sharp maximum probability for the absorption of electromagnetic radiation or capture of particles. Vibrations, frequencies and resonances are perceived by us and others as a universal phenomenon that can be shared between all living systems including biological systems and astrophysical ecosystems in away where different systems may resonate in proximity ending up with resonating together at the same frequency, to achieve a shared resonance. Striking discoveries in the vibration and resonance field is the fact that resonance frequency breathing with Heart Rate Variability (HRV) frequency around the 0.1 $\mathrm{Hz}$ peak is associated with psychophysiological well being and planetary resonance [3]. HRV is considered as a delicate and accurate measure of human Autonomic Nervous System (ANS) activity and dynamics [4] [5]. Our scientific group was able to achieve the longest record in human history of HRV synchronized to solar wind indices, Shumann Resonances (SR) and Galactic Cosmic Rays (GCR). We document that daily ANS activity responds to changes in geomagnetic and solar activity during periods of normal undisturbed activity and it is initiated at different times after the changes in the various environmental factors and persisting over varying time periods. Increase in solar wind intensity was correlated with increases in heart rate [6]. It was found that information reflecting one's emotional state is encoded in the patterns of the HRV waveform and in addition, is contained in the heart's electromagnetic field radiated into the environment [7] [8]. Alabdulgader Theory of Consciousness, The Heart Based Resonant Fields (HBRF] is based on human heart as the center of human consciousness experience that orchestrates with the planetary and cosmic energetic fields. In telephony (field of technology involved in telecommunication services for the purpose of electronic transmission of voice), the usable voice frequency band ranges from approximately 300 to $3400 \mathrm{~Hz}$. It is for this reason that the ultra low frequency band (in communication language) of the electromagnetic spectrum between 300 and $3000 \mathrm{~Hz}$ is also referred to as voice frequency, being the electromagnetic energy that represents acoustic energy at baseband. The attenuation of ELF waves is so low that they can travel completely around the Earth several times before decaying to negligible amplitude, and thus waves radiated from a source in opposite directions circumnavigating the Earth on a great circle path interfere with each other. At certain frequencies, these oppositely directed waves are in phase and add (reinforce), causing standing waves. The resonant energy of ELF in the earth ionosphere cavity is seen to be mainly dissipated in the upper wall below $56 \mathrm{~km}$ of the cavity bounded by the earth and ionosphere [9]. There is evidence on a link between the intensity of Schumann resonance measured by $\mathrm{dB}$ and environmental factors like global surface temperature [10]. Variations in the geomagnetic field (GMF) 
on the human body is said to be resonant. Decibel is useful for human perception measurement as intensity of sound and light more nearly approximates the logarithm of intensity rather than a linear relationship. The intensity of normal speech is around 60 decibel $(\mathrm{dB})$ while whisper is around $40 \mathrm{~dB}$. Human ear perceives frequencies between $20 \mathrm{~Hz}$ (lowest pitch) to $20 \mathrm{kHz}$ (highest pitch). Based on our recent Heart Based Resonant Frequencies theory on human consciousness, it seems that our consciousness as it operate in the space between our cerebral cortex and space layers is the universal great whisper [2].

\section{HEART RATE VARIABILITY FREQUENCIES: THE PSYCHOPHYSIOLOGICAL WELL BEING AND PLANETARY RESONANCES}

The understanding of Heart rate variability, defined as the change in the time intervals between adjacent heartbeats, is an emergent property of interdependent regulatory systems that operates on different time scales to adapt to environmental and psychophysiological Challenges. Understanding HRV frequencies is essential to understand the complex interacting mechanisms that operate our body systems and the surrounding near and far environment. The European Society of Cardiology and the North American Society of Pacing and Electrophysiology Task Force Report on HRV divided heart rhythm oscillations into 4 primary frequency bands; High-frequency (HF), low-frequency (VLF), very-low-frequency (VLF) and lultra ow-frequency (ULF) [3] [11]. High-frequency (HF): from $0.15 \mathrm{~Hz}$ to $0.4 \mathrm{~Hz}$, which equates to rhythms with periods that occur between 2.5 and 7 seconds. This band reflects parasympatheticor vagal activity and is frequently called the respiratory band. Low-frequency (LF): from $0.04 \mathrm{~Hz}$ to $0.15 \mathrm{~Hz}$, equates to rhythms or modulations with periods that occur between 7 and 25 seconds, This region was previously called the "baroreceptor range" or "mid-frequency band" by many researchers, since it primarily reflects baroreceptor activity while at rest. Very-low-frequency (VLF): from 0.0033 to $0.04 \mathrm{~Hz}$, equates to rhythms or modulations with periods that occur between 25 and 300 seconds and. The VLF rhythm has special peculiarity as it appears to be produced by the heart itself and may be an intrinsic rhythm that is fundamental to health and wellbeing (Figure 1). This cardiac origin of the VLF rhythm is also supported by studies showing that sympathetic blockade does not affect VLF power. Furthermore, VLF activity remains in quadriplegics, whose sympathetic innervation of the heart and lungs is disrupted [12]. The VLF band has stronger associations with all-cause mortality than LF and HF bands. Specifically, low power in this band has been associated with high inflammation and has been correlated with low levels of testosterone.

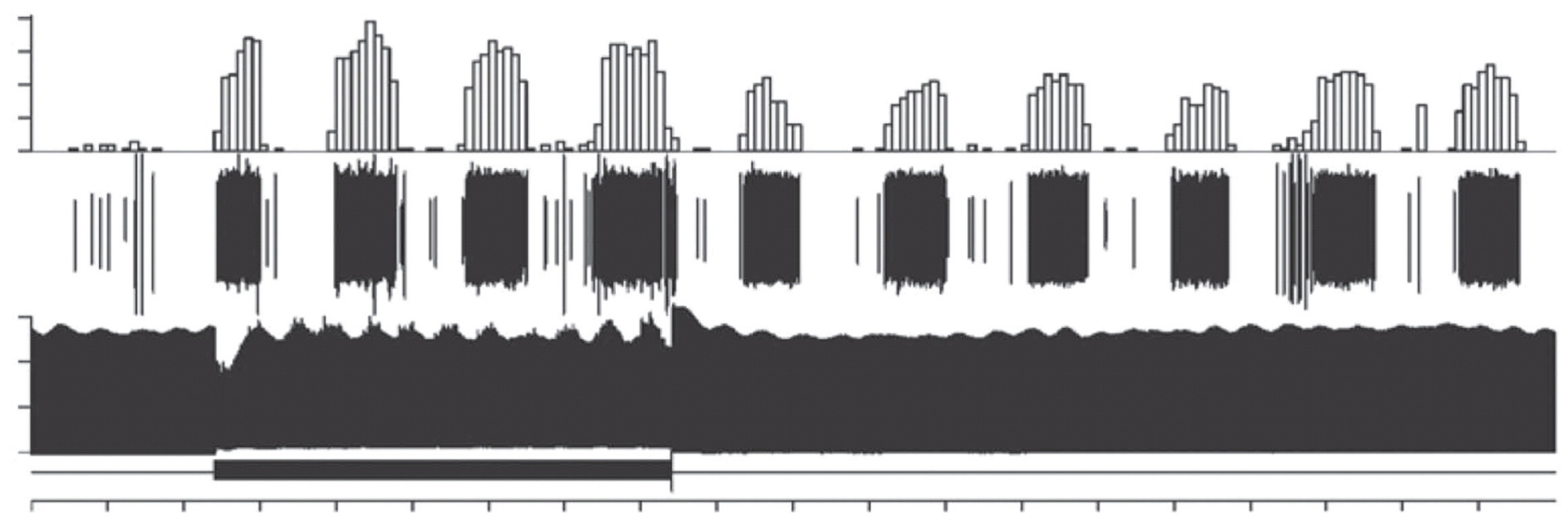

Figure 1. Long-term single-neuron recordings from an afferent neuron in the intrinsic cardiac nervous system in a beating dog heart. The top row shows neural activity. The second row is the actual neural recording. The third row is the left ventricular pressure. This intrinsic rhythm has an average period of 90 seconds with a range between 75 to 100 seconds $(0.013 \mathrm{~Hz}-0.01 \mathrm{~Hz})$, which falls within the VLF band. VLF band seems to be the subsonic internal heart whisper. Rights for Dr J. Andrew Armour. 
Ultra-low-frequency (ULF) band is below $0.0033 \mathrm{~Hz}$ (333 seconds or 5.6 minutes). The circadian oscillation in HR is the primary source of the ULF power, although other very slow-acting regulatory processes, such as core body temperature regulation, metabolism, and the renin-angiotensin system likely add to the power in this band.

The breaking ground discoveries achieved by our team in King of Organs Conferences $(2006,2008$, 2010, 2012 and 2019), HeartMath Institute and other scientific collaborators with the evidence of the universal interplay and the great symphony between human Autonomic Nervous System (ANS) represented by HRV and the cosmos is a critical scientific turning point in human history as well as, in our understanding of human species being pulsating part of endless dynamic polyverse. The heart as the dominant energetic organ of the human body and the role of heart rate variability (HRV) and its orchestrating symphony in the human body and the universe were the illuminators and the distinguished new scientific arena of the King of Organs Congresses [1]. Schumann resonances (SR) are the electromagnetic frequencies of the Earth-ionosphere cavity and were first predicted mathematically by W.O. Schumann (1952). Electromagnetic impulses, like those from global lightening flashes (the world's thunderstorm activity) fill this cavity and excite the Schumann resonances. Resonances can be observed at around 7.8, 14, 20, 26, 33, 39 and $45 \mathrm{~Hz}$ [13]. Many occupational studies have found that exposure to ELF fields between $16.7 \mathrm{~Hz}$ and $50-60 \mathrm{~Hz}$ significantly reduces melatonin levels. Similar effect to Solar Geomagnetic Activity (S-GMA) was noticed. The cell membrane has an electric field of the order of $105 \mathrm{~V} / \mathrm{cm}$. The ELF brain waves operate at about $1-10 \mathrm{~V} / \mathrm{cm}$. Fish, birds, animals and people have been shown to respond to ELF signals that produce tissue electric gradients of ULF/ELF oscillating signals at a threshold of $7-10$ to $8-10 \mathrm{~V} / \mathrm{cm}$. This involves non-linear resonant absorption of ULF/ELF oscillating signals into systems that use natural ion oscillation signals in the same frequency range [14]. Biology on earth seems to oscillate in miraculous way to those lower frequencies in a way where resulting harmonic rhythmic activities became the bases of psychophysiological well being. Perturbation of this highly organized orchestration between cosmos and biology is conceivably sensitive to changes in the solar geomagnetic activity. A hero in the field Rollin McCraty and colleagues from HeartMath Institute introduced the term physiological coherence to describe the degree of order, harmony, and stability in the various rhythmic activities within living systems over any given time period [15]. This harmonious order signifies a coherent system that has an efficient or optimal physiological functioning which will be reflected in more resilient personality and higher consciousness. Physiological coherence (also referred to as cardiac coherence) can be measured by HRV analysis where more ordered sine like HRV pattern will be seen around frequency of $0.1 \mathrm{~Hz}$ (10 seconds) which will be seen as very narrow, high-amplitude peak in the low frequency (LF) region of the HRV power spectrum with no major peaks in the VLF or HF regions [16] (Figure 2).

Mental and emotional flexibility to remain in self-directed control is a well-known outcome with repeated coherence training. This will end up with more resilient personality and psychophysiological well being including capability to reduce systemic blood pressure without medications [3] [4] [5]. It also builds one's capacity to access intuitive state with higher consciousness to achieve intelligent life options easier through what we can call heart intelligence [17].

It was found that information reflecting one's emotional state is encoded in the patterns of the HRV waveform and in addition, is contained in the heart's electromagnetic field radiated into the environment [5] [6]. There is a direct mathematical relationship between the HRV patterns and the spectral information encoded in the magnetic field [5]. Karl Pribram's who theorized the holonomic mind brain theory proposed that low frequency oscillations generated by the heart and body in the form of afferent neural hormonal and electrical patterns are the carriers of emotional information and the higher frequency oscillations found in the EEG reflect the consciousness. Human species are not in isolation from the near and far environments and ecosystems. All biological systems on the planet are exposed to an external and internal environment of fluctuating invisible wide range of magnetic fields and frequencies. These fields can function as information carrier and being pattern specific can affect virtually every cell and circuit to a greater or lesser degree. Numerous physiological rhythms including cardiovascular system, and nervous system have been shown to be synchronized with solar and geomagnetic activity [6]. Geomagnetic and solar 


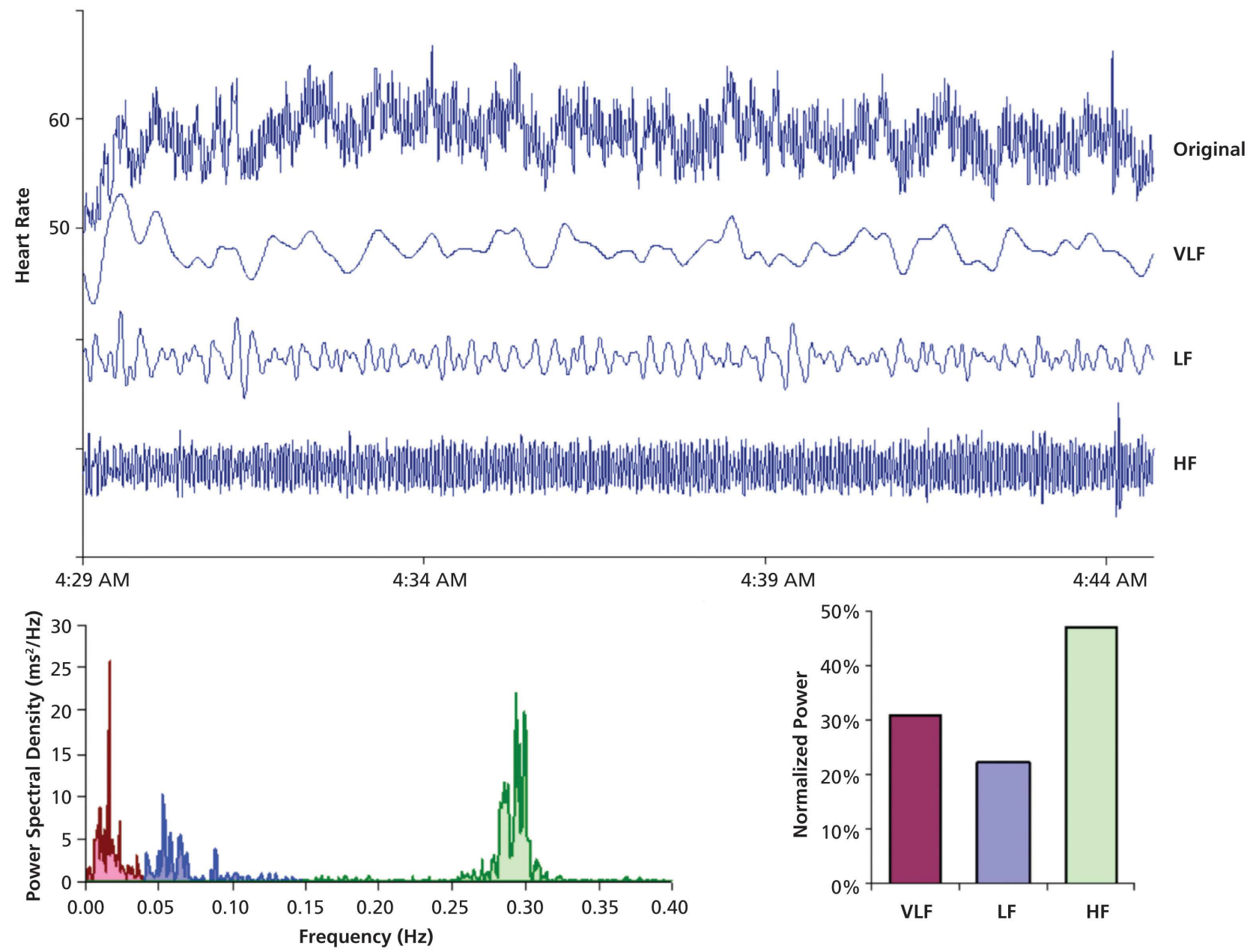

Figure 2. The top tracing shows the original HRV waveform. Filtering techniques were used to separate the original wave form into VLF, LF, and HF bands as shown in the lower traces. The bottom of the figure shows the power spectra (left) and the percentage of power (right) in each band. abbreviations: HF, high frequency; LF, low frequency; VLF, very low frequency; PSD: power spectral density.

influences affect a wide range of human rhythmic systems with the nervous and cardiovascular systems, with their significant contribution to consciousness, being the most clearly impacted [3]. We adopt holistic visionary perspective implies that level of human consciousness is the collective activity of widespread areas of bilateral association of cortical and subcortical structures and other interconnected biological and astrophysical systems. Alexander Leonidovich Tchizhevsky (1897-1964), founder of cosmic ecology and heliobiology, followed by Franz Halberg (1919-2013) and our team in the King of Organs Conferences, HeartMath Institute (HMI) and the Global Coherence Initiative (GCI) established the scientific prove of earth magnetic field correlation to biology in earth. The evidence of the resonance between the biology of human being as represented by HRV and the cosmos is a major turning point in the scientific and conceptual understanding of the humanity (Figure 3). The spectrum of the operating frequencies of the solar wind speed, Kp, Ap, solar radio flux, cosmic ray counts, Schumann resonance power, and the total variations in the magnetic field with its overlapping frequency spectrum with human heart and autonomic nervous system (ANS) and with biology in earth seems to be the miraculous whisper that operate our consciousness in earth [18]-[23]. 

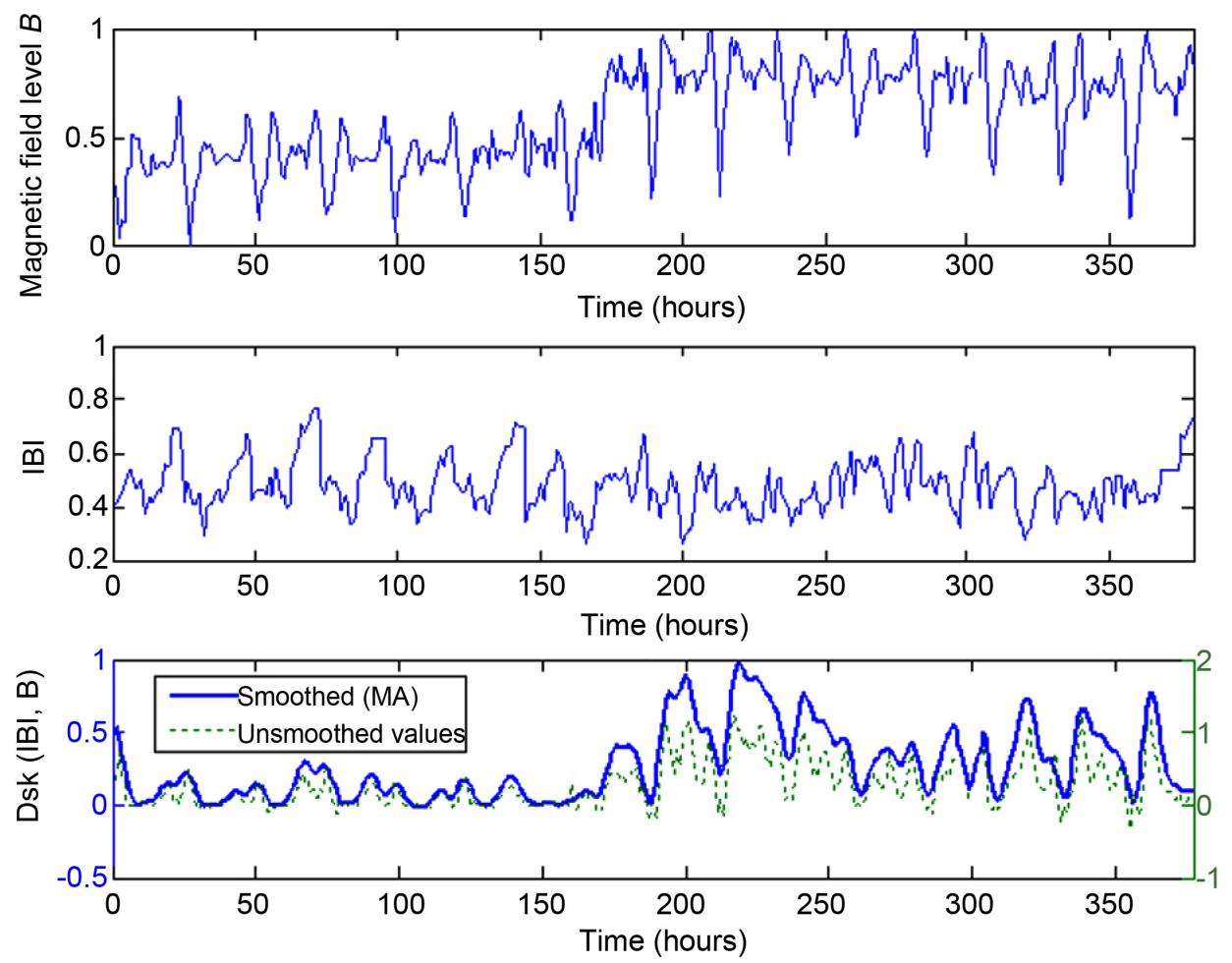

Figure 3. Our local record of earth MF and heart rate variability. HRV synchronous fluctuation to local magnetic field level is shown. (IBI: Inter Beat Interval).

\section{THE BEATING HEART VAGAL AFFERENTS, THE EARTH HEART BEAT (SR) AND BEYOND: THE HUMANITY INTERCONNECTEDNESS AND THE WHISPERING UNIVERSE}

It is well known that the cardiovascular system of the human body has the ability to generate electromagnetic fields with a main proper frequency in the range of 0.8 to $2.5 \mathrm{~Hz}$ [24]. The amplitude of the cardiac electrical signal is about 60 times greater compared to the brain while heart electrical field is 100 times stronger than the brain electrical field and the electromagnetic field of the heart is approximately 5000 times stronger and can be detected six feet away from the body with sensitive magnetometers [1] [13]. The real contribution of human brain in consciousness experience, although being important is not at all exclusive Eben Alexander, is an American neurosurgeon who authored Proof of Heavenbook, where he described his personal experience, asserts that consciousness is not only a product of the brain. In historical appraisal R. Nitsch and F. W. Stahnisch in the journal Cerebral Cortex challenged Penfield original concept of experiential phenomena elicited by electrical Stimulation of the human cortex. They revisited Penfield clinical work and found that the actual results obtained from electrical stimulation studies of the brain are far less conclusive [25] [26]. In consciousness science new scientific findings of the elegant role of the afferent vagal nerve has been discussed in many publications by us and others in the last 4 decades. The majority of higher brain centers, as well as emotional experience and cognitive processes are operated by Cardiovascular related afferent neural traffic [1] [7].

An important land mark in the field was John and Beatrice Lacey observations during 1960s and 1970s that important consciousness pillars such as perception, cognitive and behavior are affected significantly by afferent input from the heart and cardiovascular system [27]. Laceys showed that patterns of physiological responses were affected as much by the context of a specific task and its requirements as by emotional stimuli. A phenomenon called by Laceys the directional fractionation denotes paradoxical heart rate response as it decelerated and blood pressure decreased, while simultaneously recorded parameters 
such as, respiratory rate, pupillary dilation and skin conductance all increased as expected [28]. Later, cognitive performance fluctuated at a rhythm around $0.1 \mathrm{~Hz}$ was demonstrated by Velden and Wolk and showed that the modulation of cortical function via the heart's influence was due to afferent inputs on the neurons in the thalamus, which globally synchronizes cortical activity and the consciousness phenomena [29] [30]. Rollin McCraty in the HeartMath Institute coined the term heart coherence (also referred to as cardiac coherence or resonance) which can be measured by HRV analysis wherein a person's heart rhythm pattern becomes more ordered and sine-wave like at a frequency of around $0.1 \mathrm{~Hz}$ (10 seconds). A coherent heart rhythm is defined as a relatively harmonic, sine wave-like, signal with a very narrow, high-amplitude peak in the LF region of the HRV power spectrum with no major peaks in the VLF or HF regions [15]. HRV sub analysis is highly delicate tool to explore in human different emotions (Figure 4). It seems that the different peaks of the different frequencies are the subsonic whisper of our collective consciousness that resonate in the universal hierarchy to impact the global energetic fields.
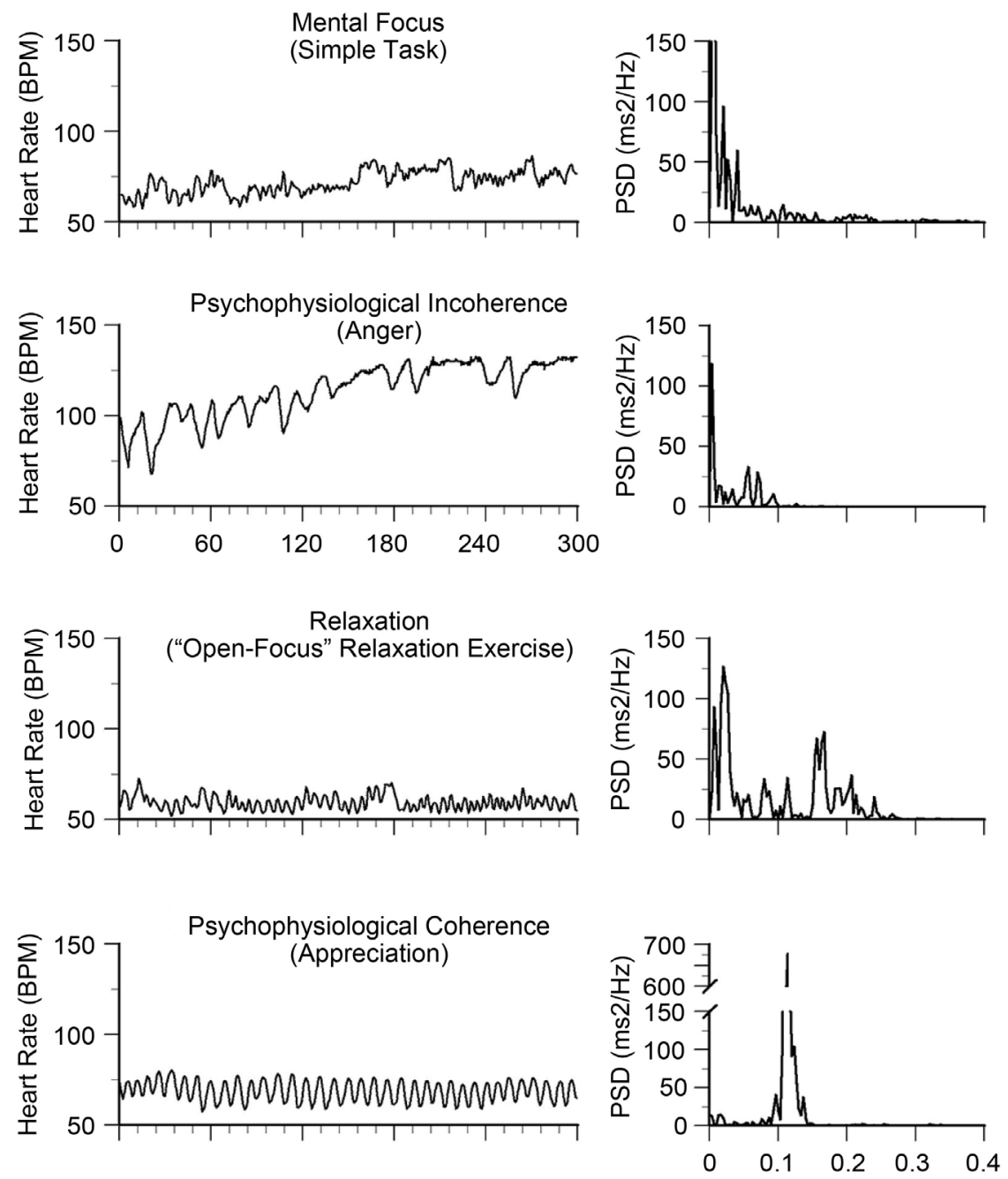

Figure 4. HRV waveforms typically observed in differing psychological states. Psychophysiological coherence is represented by sine wave like, signal with a very narrow, high-amplitude peak around $0.1 \mathrm{~Hz}$ in the LF region of the HRV power spectrum with no major peaks in the VLF or HF regions. The power spectral density (PSD) analysis of the HRV rhythms for each emotion is shown on the left. 
A critical observation here is the finding that "pattern and stability" (of the rhythm) of the heart's afferent inputs, rather than the number of neural bursts within the cardiac cycle that will modulate thalamic activity, which in turn has global effects on brain function and ultimately the consciousness experience [3]. Activating afferent input with vagal nerve stimulation (VNS) is apparently heralding a new era in medical therapeutics as it proves effective in many psychophysiological disorders including epilepsy, obesity, depression, anxiety, autism, alcohol addiction, mood disorders, as well as multiple sclerosis, and traumatic brain injury [31] [32]. The cardiac coherence training is known to intensify afferent vagal neuronal inputs to cortical and subcortical systems and to Neuronal Correlates of Consciousness (NCC) with long term capabilities to reset the reference set up points resulting in increased afferent nerve activity noninvasively and ultimately improves psychophysiological parameters and the consciousness experience. There is thus a need to explore novel ways of repairing lost consciousness. Vagus nerve stimulation (VNS) may contribute to breaking advances in awakening the unconscious vegetative state patient as approved by improvement in behavioral responsiveness and enhanced brain connectivity patterns. An important finding with significant contribution to heart neuronal input to the brain is that vagus nerve carries afferent connections to the deep nuclei of the brain via the nucleus solitaries. These afferent connections have multiple consciousness related targets, which include the thalamus, amygdala, reticular formation, hippocampus, raphe nucleus, and the locus coeruleus. VNS will create improved global neurostimulation state leading to promoted spread of cortical signals and caused an increase of metabolic activity leading to behavioral improvement as measured with the Coma Recovery Scale-Revised (CRS-R) scale [33]. Theta waves dominance were shown in the right inferior parietal and the parieto-temporal-occipital border, a region known to be instrumental in conscious awareness. Improvement in long white matter tracts namely the corticocortical and thalamocortical disconnected pathways by vagus nerve stimulation is a true revolutionary therapeutic option.

Today it is conspicuous to the whole medical communities that vagal nerve is never vagal. It is a critical component of the great universal loop of human consciousness including human heart, brain and the resonant universal fields with its universal great hierarchy. The symphony of the highly orchestrated signaling and frequencies as measured from HRV reflecting human autonomic nervous system activity, not only responds to changes in solar and geomagnetic activity, but is synchronized with the time-varying magnetic fields associated with geomagnetic field-line resonances and Schumann resonances. It is found that the Schumann Resonance signal is extremely highly correlated with S-GMA indices of sunspot number and the Kp index. The SR signal is a globally available signal that has been generated since the ionosphere was formed and thunderstorms have existed. Hence they pre-date animal evolution on earth .The physical mechanism was thought to be related to activity of the ionospheric D-region ion/electron density that varies with S-GMA and forms the upper boundary of the resonant cavity in which the Schumann Resonance signal is formed [14]. The first five modes [7] [8] [14] [20] [26] [33] Hz dominated the daytime. At night their intensity and frequency decreased and a large proportion of signals were found to be less than $4 \mathrm{~Hz}$. This frequency and intensity shift is from increasing the depth of the resonant cavity in the nocturnal hemisphere. The ion/electron density in the D-Region decreases rapidly after sunset as the solar production of ions ceases and recombination dominates [14]. Our scientific group most plausible opinion to explain how solar and geomagnetic fields can influence human nervous system activity is through a resonant coupling between our nervous systems and geomagnetic frequencies (Alfvén waves), or ultra low frequency standing waves in the earth-ionosphere resonant cavity (Schumann resonances) that overlap with physiological rhythms [19]. The Global Coherence Initiative (GCI) foundation is a land mark in the scientific history of helibiology. It is the first global network of GPS time stamped detectors designed to continuously measure magnetic signals that occur in the same range as human physiological frequencies such as the brain and cardiovascular systems. Each site includes ultrasensitive magnetic field detectors (sensitivity $10^{-12} \mathrm{~T}$ ) specifically designed to measure the magnetic resonances in the earth/ionosphere cavity, resonances that are generated by the vibrations of the earth's geomagnetic field lines and ultra-low frequencies that occur in the earth's magnetic field, all of which have been shown to impact human health, mental and emotional processes and behaviors. Each monitoring site detects the local alternating magnetic 
field strengths in 3 dimensions over a relatively wide frequency range $(0.01-300 \mathrm{~Hz})$ while maintaining a flat frequency response [3]. Our research in the last two decades is based on the believe that heart electromagnetic field and the different HRV patterns are a delicate information carriers. An energy-based concept of information is not new as it was described by Dennis Gabor, who invented the hologram in 1947, which he described as quanta of information. As a matter of fact, there is a direct mathematical relationship between the HRV patterns and the spectral information encoded in the magnetic field [7]. The coherence model predicts that different emotions are reflected in state-specific patterns in the heart's rhythms regardless of the heart rate. Patterns in the activity of cardiovascular afferent neuronal traffic can significantly influence cognitive performance, emotional experience and self regulatory capacity via inputs to the thalamus, amygdala and other subcortical structures [1].

It was shown that when two people are in a loving relationship that their hearts rhythms can synchronize even at a distances of 8000 kilometers (Peter Granger presentation in King of Organs 2019). We just concluded a Global study of human heart rhythm synchronization with the Earth's time varying magnetic field. During a 15-day period, 104 participants located in Saudi Arabia, California, Lithuania, New Zealand, and England underwent continuous ambulatory HRV monitoring. The local time varying magnetic field (LMF) intensity was obtained using a time synchronized and calibrated network of magnetometers located at the five monitoring sites in the same geographical locations as the participant groups. Changes in geomagnetic conditions have been shown to affect the rhythms produced by the brain and heart and that human autonomic nervous system activity reflected in heart rate variability (HRV) over longer time periods can synchronize to changes in the amplitude of resonant frequencies produced by geomagnetic field-line and Schumann resonances. The only day where all the groups HRV was positively correlated with the local time varying magnetic field (LMF) was on the day of the Heart Lock-In (HLI) which has been shown to increase the coherence in one's heart rhythms as well as increase the synchronization of heart rhythms between participants in a group setting -for a 15-minute period and the synchronization between the HRV and local time varying magnetic field (LMF) for all the groups was significantly higher than most of the other days [23].

\section{HEART BASED RESONANT FREQUENCIES, WHISPERING UNIVERSAL VIBRATIONS AND THE HUMAN CONSCIOUSNESS}

The history of human being in earth over the diverse civilizations has been linked to space since antiquity until today. The sun and the solar system is the most remarkable and attractive part of the space affecting human life since creature. The sun is the humanity universal symbol for energy and life on earth and was considered to be in connection with the source of energy and life organ of the human being, namely, the Heart. The new era of advances in biology, astrobiology and space sciences witnessed new understanding at clear intersection with the ancient wisdom. On the other hand, the new understanding of the human Heart, Heart Rate Variability (HRV) and its cosmic connections create revolutionary era with its expected impact on the practice of medicine. The sun is known to fuse about 600 million tons of hydrogen into helium every second, converting 4 million tons of matter into energy every second as a result. This energy, which can take between 10,000 and 170,000 years to escape the core, is the source of the Sun's light and heat with its consequences on life in the planet. During Solar flares an energy release of $10^{20}-10^{25}$ joules is emitted. This gigantic star with its flares and spots attracted the attention of many scientists over human history, but the Russian biophysicist who established modern heliobiology, Alexander Leonidovich Chizhevsky was special land mark in the field. The heart and its gigantic communication capabilities attracted the attention of our scientific group in Heart Math Institute since 1991 and King of Organs Conferences (2006, 2008, 2010, 2012 and 2019), headed by the author, and our scientific group including Franz Halberge the father of modern chronobiology, RollinMcCraty, Germaine Cornelssen and other heroes in the field. This astonishing scientific group of scientist in the modern era sparkled by $A$ L Chizhevsky established a genuine true scientific connection between the two great miraculous creatures: the human Heart and the Sun. 


\section{A L Chizhevsky, GeoManetic Activity (GMA) and Mass Human Events}

Alexander Leonidovich Chizhevsky (7 February1897-20 December 1964) the extraordinary student of the Moscow Archaeological Institute in 1915, is the father of heliobiology in the modern ages. We considered ourselves, as scientist in the science of cosmic and biological unity, the second generation of our scientific father A L Chizhevsky. Chizhevsky's ideas about unity of human, terrestrial and cosmic processes were met with sharp rejection. In 1935, the newspaper Pravda published an article entitled "The Enemy under the Mask of a Scientist", in which Chizhevsky was accused of counter-revolutionary activity for which he was arrested in January 1942 and sentenced to eight years for anti-Soviet activity. Today's astrophysicists figuratively say that we all live in the Sun's atmosphere, and our lifes depend on its "weath$e r$ " changes. Respectful scientific literature nowadays witnessed the novel historical observations documented by the father of the field, A L Chizhevsky who established the relationship of cosmophysical factors to the dynamics of social processes and mass humanity events. He summarized his statistical research in a his illustration tabel covering 2500 years of human history which he calls "Histrometrical table for the period from the Fifth Century B.C to the Twentieth Century A.D". Chizhevsky's established the cyclicity of years over epochs of time where each solar cycle equals 11 years. The 11 years cycle is subdivided to Minimum of Excitability: 3 years, Growth of Excitability: 2 years, Maximum of Excitability: 3 years, Decline of Excitability: 3 years [34]. The Chizhevsky's Histotrometric documentations is in our views a significant land mark in human history alerting humanity to the cosmophysical effect on global humanity consciousness (Figure 5).

The sun's magnetic field, called the interplanetary magnetic field (IMF), extends far out into space, beyond the farthest planet. The region of space the IMF extends into is called the heliosphere, and it acts as a magnetic shield for our entire solar system against cosmic rays, which are charged particles from deep space. The solar wind, which is the stream of charged particles that flows outward from the sun, travels at an average speed of around 1 million miles per hour. Solar wind interacts with planetary magnetic fields in complex ways leads to "field-line resonances" in the earth's magnetic fields. For now, it should be noted that during solar storms the solar wind speed can increase dramatically to a speed around 5000 miles per hour and cause disruptions in the earth's magnetic field. Earth's magnetic field, also known as the geomagnetic field, is the field that extends from the earth's interior to where it meets the solar wind. This region is called the magnetosphere. Every cell in every biological system on Earth is bathed in an external and internal environment of fluctuating invisible magnetic fields of a wide range of frequencies, these fields can affect virtually every cell and circuit in biological systems [35]. During periods of increased solar activity, which peaks every 10.5 to 11 years, the sun emits increased ultraviolet (UV) energy and solar radio flux, which is measured by the $2.8 \mathrm{GHz}$ signal (F10.7) radiated by the sun [35] [36] [37]. A coronal mass ejection $(C M E)$ is a significant release of plasma and accompanying magnetic field from the solar corona. They often follow solar flares and are normally present during a solar prominence eruption. The solar coronal ejection radiation reaches earth in 8 minutes and the electromagnetic radiation lead to sharp reorganization of the ionosphere current systems. This leads to sharp increase of about 10 nano Tesla of the variable magnetic field. An example of our record of the magnetic field and the disturbance created by CME is shown (Figure 6). A thorough search for a factor within the magnetic field fluctuations showed a correspondence of the human reaction with the occurrence of intensive long period ( 2 - $240 \mathrm{~min})$ fluctuations of geomagnetic field prior to the beginning of magnetic storms. These fluctuations are caused by oscillations of solar wind plasma and, in some cases, are connected to fluctuations in active areas of the Sun, which occur before solar flares. The reaction of live organisms to fluctuations of magnetic field with particular periodicity can be explained by the theory of parametric resonance in biological systems proposed by Lednev based on an earlier atomic spectroscopy theory described by Podgoretskii and Khrustalev. The ion parametric resonance (IPR) model developed by Jbblanchard and CF Blackman corrects mathematical errors in the earlier Lednev model and extends that model to give explicit predictions of biological responses to parallel AC 40 and DC magnetic fields caused by field-induced changes in combinations of ions within the biological system [38]. Fluctuations with the periods range of $2-240$ min can influence the 


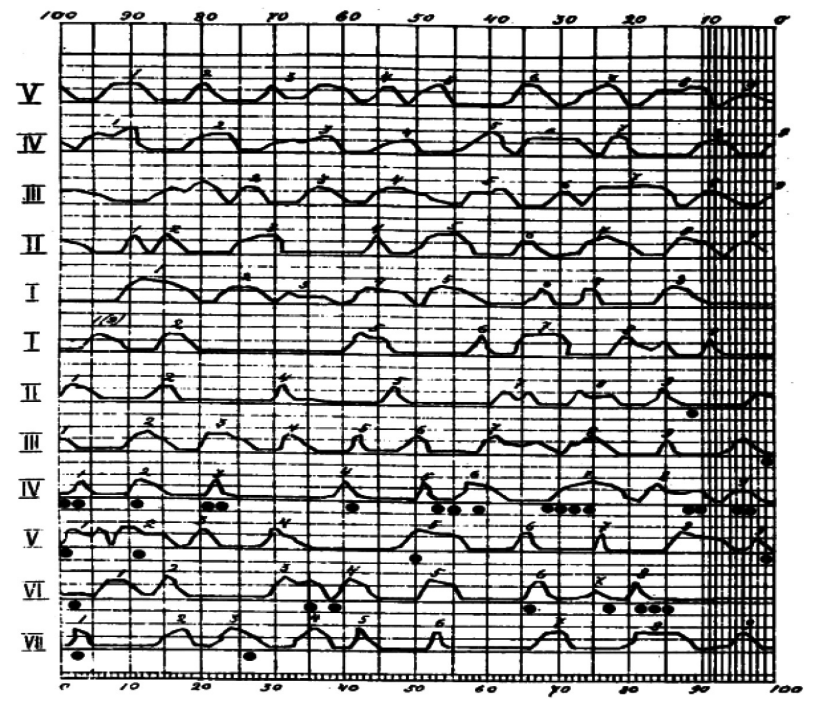

(a)

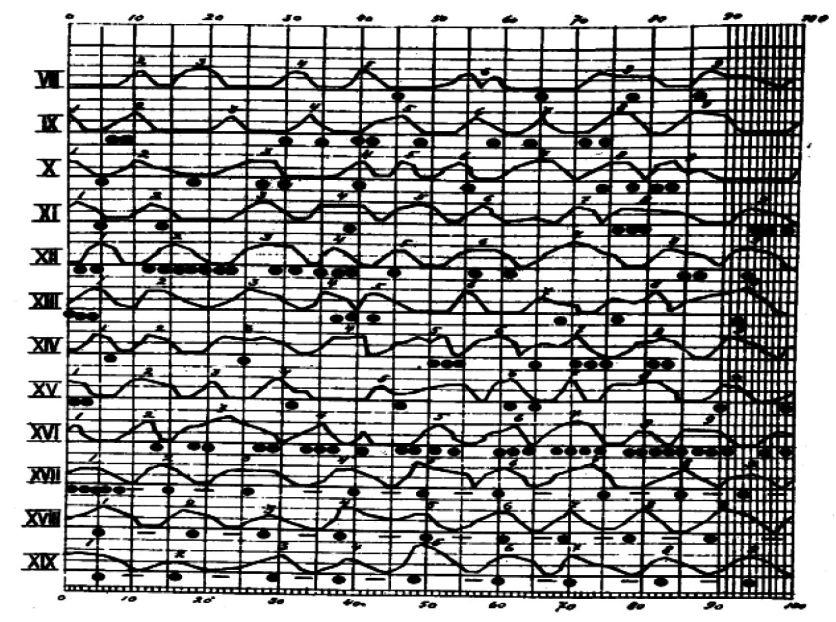

(b)
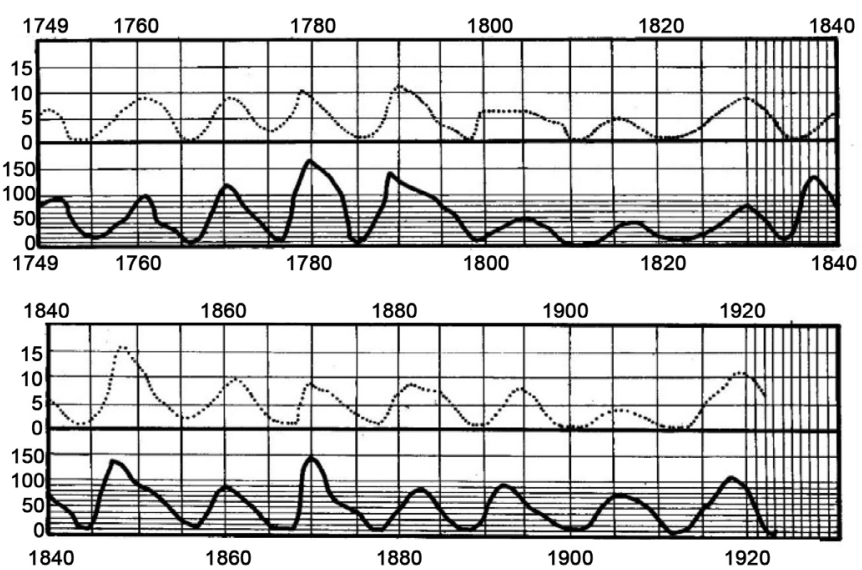

(c)

Figure 5. Chizhevsky original graphs of the universal historical process in all the surface of the earth over 2500 years. Courtesy: Sergey smelyakov. Tchijevsky disclosure: how the solar cycles modulate the history. http://www.ASTROTHEOS.COM 


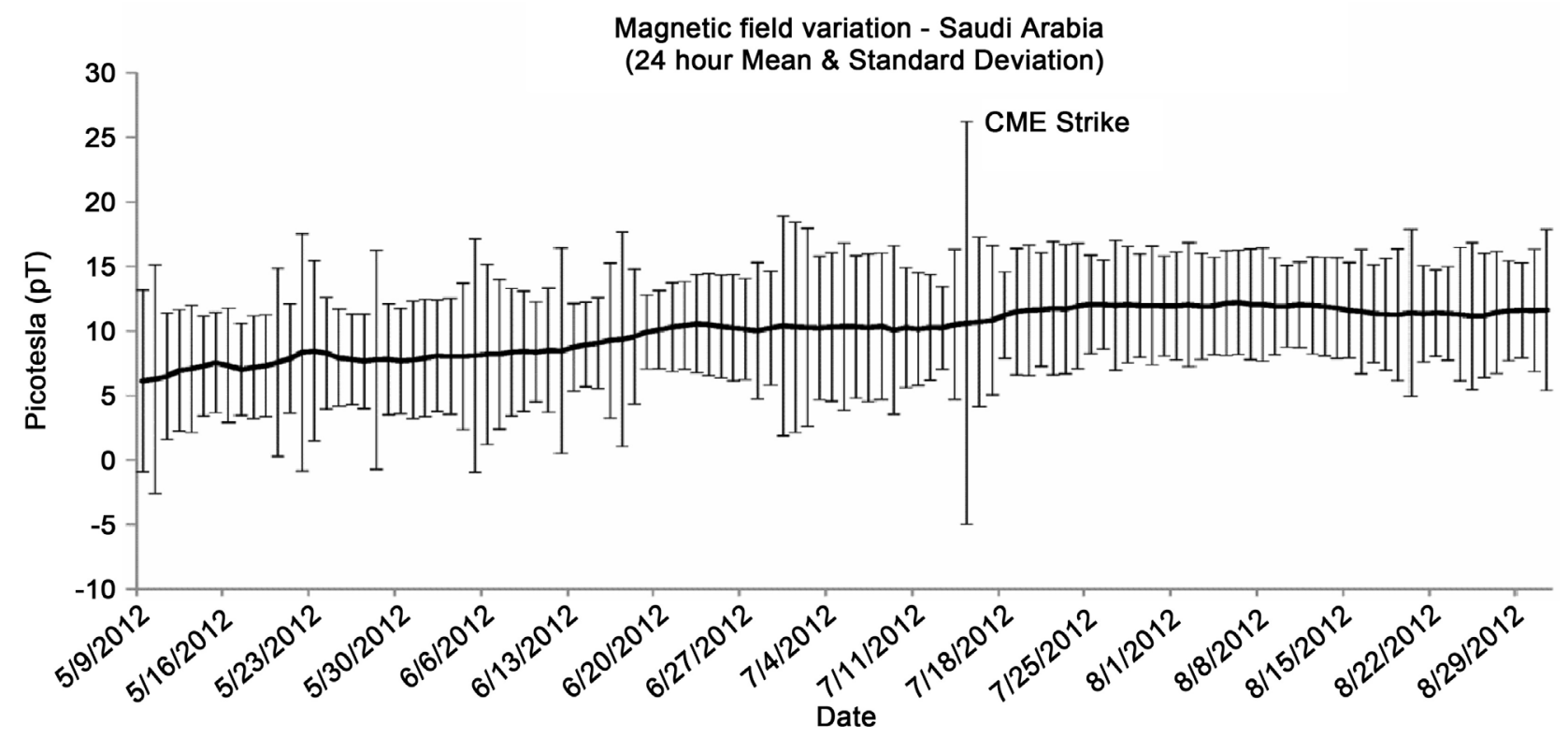

Figure 6. Mean and standard deviation of the magnetic-field data recorded from the Saudi Arabia monitoring site.

brain and the endocrine system and initiate a "hypothalamus-hypophysis-adrenal gland" circuit, which lead to a stressful condition. This phenomenon is referred in the concerned literature as The Tchizhevsky-Velhover effect, or the effect of "anticipatory reaction" of biological entities [39]. The sporadic stream of plasma of the solar wind reaches the earth in 1 - 2 days and as a result of its interaction with the magnetosphere a magnetic storm begins [40].

In a review of the research literature on health effects of geomagnetic disturbances, Palmer, Rycroft and Cermack observed these "definite conclusions": 1) Geomagnetic disturbances have a greater effect on humans at higher geomagnetic latitudes. 2) Unusually high values of geomagnetic activity have an effect on human cardiovascular health. 3) Unusually low values of geomagnetic activity seem to have an effect on human health. 4) Only $10 \%$ to $15 \%$ of people in areas studied are significantly affected by geomagnetic activity. 5) HRV is negatively correlated with geomagnetic activity [35] [40].

\section{HUMAN CONSCIOUSNESS: THE UNIVERSAL HEART BASED RESONANT FREQUENCIES AND THE ECOSYSTEM VIBRATIONS: THE VENERABLE WHISPER}

Human consciousness has been elusive scientific and spiritual issue since the dawn of humanity. The birth of quantum physics as distinct branch of physics which stands alone as unique human understanding with its probabilistic and non locality back ground creates breakthrough in humanity understanding of the astrophysical phenomenon and the elusive biological events such as consciousness experience. In addition, influx of new knowledge at the end of the 20th century sheds new lights and contributes significantly to the process of decoding of human mysteries in the universe. Parallel progress in cosmology, geology, ecology and biology in the 1960s and 1970s hasmodified our ideas about the universe, and the human conscious experience. But these contributions remain somewhat incoherent and disjointed. The pieces of the puzzle need to reconstruct to show the expected spectacular image. There is impressive progress in knowledge of the parts and regrettable paradoxical ignorance of the whole for which integral universal model is in immediate demand.

The idea that quantum mechanics has something to do with the workings of the mind was developed by Eugene Wigner, Hungarian-American theoretical physicist and Nobel Prize Laurete in Physics in 1963, who proposed that the wave function collapses due to its interaction with consciousness [1] [2]. Roger 
Nelson and Helané Wahbeh from the Global Consciousness Project (GCP) provided recent evidence supporting the hypothesis that our consciousness can even influences our physical world. They adopt Random number generators (RNGs) as a tool used to evaluate micro-psychokinesis or our ability to affect the physical world with our consciousness. Roger Nelson reported in a recent publication multiple examples of striking similarity between event-related brain potentials and event-related correlations in random data (Figure 7) [41].

Quantum physicists Larissa Brizhik and Emilio DelGiudice suggested that the most likely physical agent that can continuously provide an exchange of information between living systems within the larger ecosystem is the magnetic fields. They postulate that electromagnetic potential can cause the emergence of the coherent structures that in view of their coherence, openness and non-linearity, are able to self-organize and form various hierarchical levels of ecosystems [42]. The quantum field theory, denotes that potentials of the magnetic field, governs the dynamics of biological systems and the whole ecosystem. David Bohm's views perceived mind and matter as projections into our explicate order from the underlying implicate order. Bohm's "Wholeness," might be extrapolated to establish a direct connection between spirit and consciousness through the phenomenon of frequency resonance operating through Fourier transform-like mathematical processes, where spirit is the non local phenomenon while human consciousness is the local event. An energy-based concept of information was described by Dennis Gabor in 1947. Later on, he won Nobel prize in physics for this invention in 1971. Karl H. Pribram'sholonomic model of brain processing was described in his 1991 Brain and Perception book which include his perspectives on human consciousness with David Bohm. It describes human cognition by modeling the brain as a holographic storage network. Pribram suggests these processes involve electric oscillations in the brain's dendritic networks, which are different from the more commonly known action potentials involving axons and synapses. Pribram's holonomic model contributes significantly to human consciousness understanding, specially to the fast associative memory and the non-locality of memory. In 1991 Roger Penrose who is Nobel laureate for physics and Stuart Hameroff who is anesthesiologist and psychologist introduced the Orchestrated Objective Reduction (Orch-OR) theory which is a biological philosophy of mind which postulates that at the quantum level consciousness originates inside neurons, rather than the traditional perspective that it is a product of connections between neurons [2]. Their mechanism is based on non computational quantum process performed by quantum bits [qubits] formed collectively on cellular microtubules called objective reduction. In 1967 Hiroomi Umezawa and collaborators proposed a quantum field theory of memory storage which is fundamentally different from the Penrose-Hameroff theory based on quantum

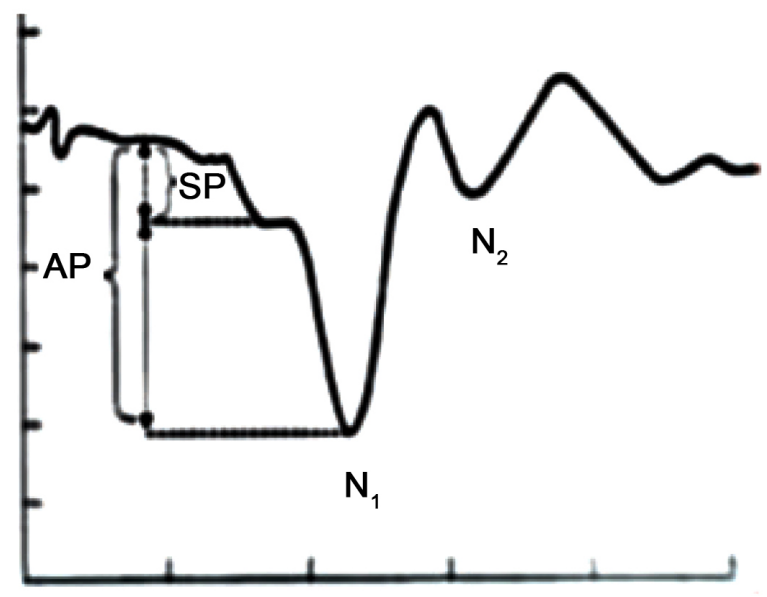

Composite of 9 Significant 6 hour Events

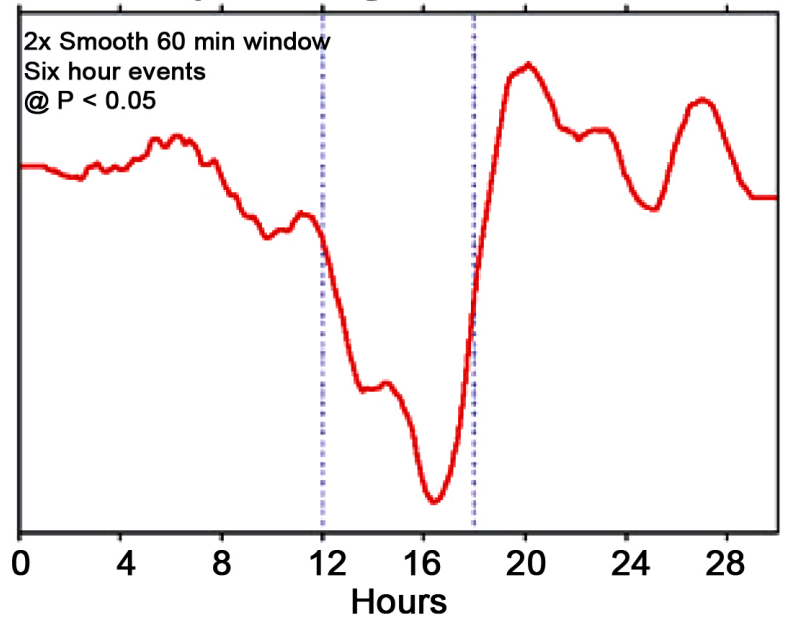

Figure 7. Evoked Potential (EP) waveform from normal electrocochleaogram (OCoG), displays signal-averaged data from electrodes placed trans-tympanically into the cochlea (the black) showing striking resemblance to composite of GCP data waveform from nine 6 hour events (the red) [41]. 
theory of the brain which posits a spatially distributed charge formation exhibiting spontaneous breakdowns at micro levels as the basis for processing at macro levels. According to this model, the information resides in the virtual field associated with the dynamics of the cellular matter. The approach of Hiroomi Umezawa was built upon by Karl Pribram and many others and expanded by Giuseppe Vitiello to a dissipative quantum model of brain. Henry Pierce Stapp postulates a process of global collapse through an effect on the synapses by exploitation certain aspects of quantum Zeno effect. Each theory discussed has its strength and weaknesses but all lack the comprehensive universal perspective incorporating the origin of consciousness with more homogenous incorporation of current theories in a stronger model capable of bringing us closer to consciousness realm closer than any time ever.

It is imperative at this time of scientific history to emphasize in critical conceptual difference between our work in the field and our theory of consciousness compared to all previous theories and models. All previous theories were based on the undebatable understanding that human brain is the source of the human consciousness experience. We recently published an elaborated chapter on the role of non cerebral cortex role in human consciousness entitled "Human Consciousness: The role of Cerebral and cerebellar Cortex, vagal afferents, and Beyond" [1]. Our theory (Alabdulgader Theory of Consciousness, The Heart Based Resonant Fields (HBRF)) is based on human heart as the center of human consciousness experience that orchestrate with the planetary and cosmic energetic fields. We were able to achieve the longest record of human Heart Rate Variability (HRV) (96000 hours) synchronized with Schumann Resonances (SR), Solar Wind indices and the extremely far Cosmic frequencies originating from the endless universal nuclear explosions, namely the Galactic Cosmic Rays (GCR) [6]. Our ultrasensitive magnetic field detectors consist of induction coil magnetometers (Zonge ANT-4; sensitivity $10-12 \mathrm{~T}$ ) as part of the Global Coherence Initiative (GCI) Project. Our site includes three magnetometers positioned in the north-south, east-west axis and vertical to detect local time varying magnetic field strengths over a relatively wide frequency range $(0.001-50 \mathrm{~Hz})$ while maintaining a flat frequency response. The data acquisition infrastructure collects and timestamps all data using GPS time signals before uploading to a common server. We documented that daily Autonomic Nervous System (ANS) activity responds to change in geomagnetic and solar activity during periods of normal undisturbed activity and it is initiated at different times after the changes in the various environmental factors and persist over varying time periods. Increase in solar wind intensity was correlated with increases in heart rate, which we interpreted as a biological stress response. Increase in Cosmic Rays, Solar radio flux, and Schumann Resonance power was all associated with increased HRV and parasympathetic activity. We have shown that energetic universal vibrations and frequencies at subsonic level whisper in a delicate manner and orchestrate our conscious experience in different ways depending on subjects sensitivity, health status and capacity for self-regulation [6] (Figure 8 and Figure 9).

There is a direct mathematical relationship between the HRV patterns which contains our magnetic potentials of our emotions and the spectral information encoded in the magnetic field [41]. This ultimately means that our emotions and consciousness are frequencies that pulsate and resonate with the far universe in highly organized hierarchical manner. Electrosolitons, which are the charge, energy, and information carriers in the metabolic processes in biological systems, according to Davydov's hypothesis [37], are formed in alpha-helical proteins, DNA, and hydrogen-bonded water molecule chains, present in living cells, intercellular tissues, and in all ecological systems. The electromagnetic fields of the universal systems and its associated potential patterns are the information carrier. The dynamic mass of the electrosoliton depends on the frequency of the magnetic field which affects charge transport processes. At the resonant frequency, electrosoliton wave will be drifted by the magnetic field and accordingly emit radiation and whisper in the space with sound waves intensively then lose their energy. Electrosolitons emit their own electromagnetic radiation with the frequency of the main harmonic which is dependent on their own velocity. Electromagnetic potential can cause the emergence of the coherent structures that in view of their coherence, openness, and nonlinearity, are able to self-organize and form various hierarchical levels of ecosystems [40]. Via this electrosoliton emitted electromagnetic radiation, subunits of the complex system (cells, organs, individuals, ecosystems) exchange their information which leads to the appearance of the 


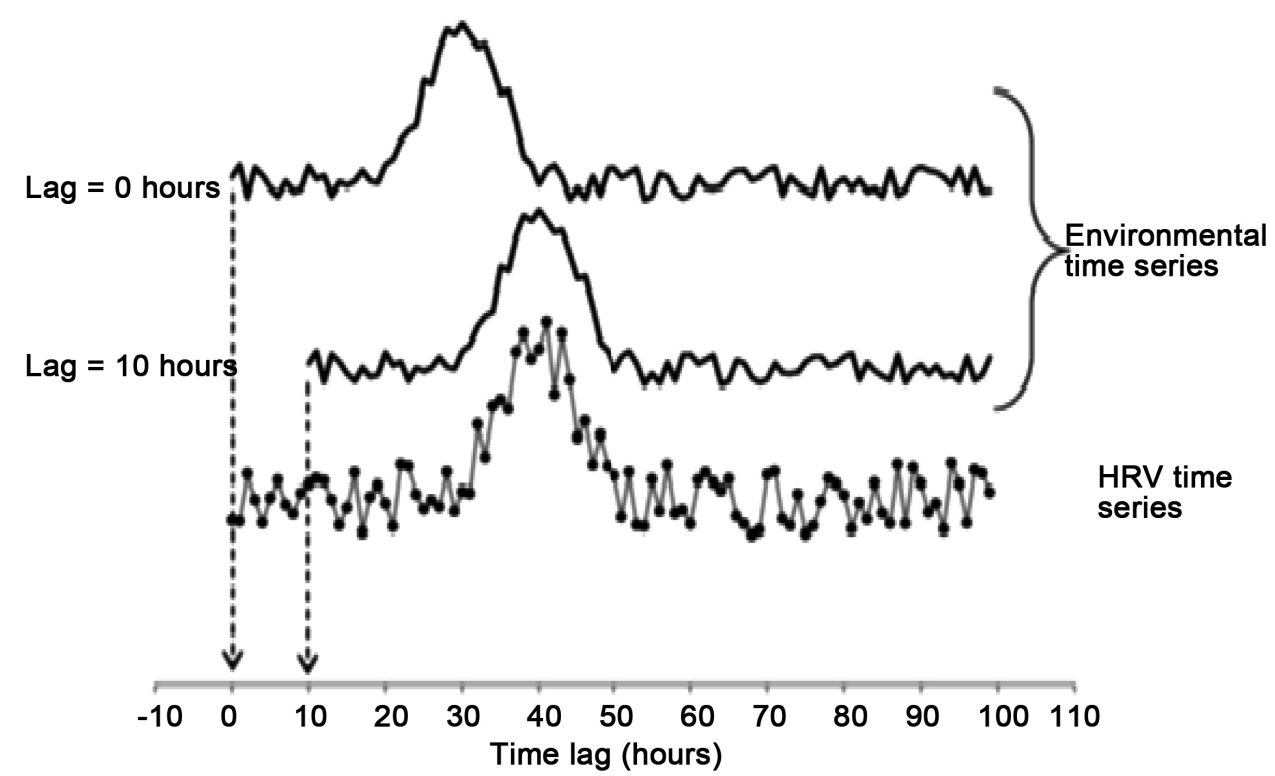

Figure 8. Our data series representing an environmental time series and a HRV time series. The environmental signals position at lag 0 (upper series) is as it occurred relative to the HRV signal. The middle trace shows the same environmental data series after shifting it in time by 10 hours. In this example, correlations between the HRV and environmental data would be higher at lag 10 than at lag 0 indicating a delayed physiological response to the external environmental signal (Tchizhevsky-Velhover effect, or the effect of "anticipatory reaction" of biological entities).

long-range order in a process of the massive hierarchy of ecosystems that is ongoing in descending manner from the galaxies to the local electromagnetic field (EMF) of the heart and brain with impressive accuracy. The pulsating electromagnetic power of the human heart with its marvelous HRV frequencies is the carries of our conscious experience on earth. The human heart delicate resonance with the universe is seemingly the great whisper of our spirits to the highest power ever. In our domain we call him ALLAH.

\section{CONCLUSION}

Human consciousness has been elusory secret for humanity over the different civilizations. Intuitive knowledge of consciousness in the ancient wisdom points to the compulsive contribution of sun and space in human physical as well as spiritual life. The refute of the twentieth century neural firing theory is a restore of the confidence of thousands of years of human civilizations intuiting the role of sun and space in human consciousness. Paucity of true comprehensive experimental work and dominance of theoretical and philosophical theories in the field is the role. The concept of resonance and its implementations in physiological as well as astrophysical rhythms is of critical significance for life on earth and to human consciousness experience. Holistic perspective of human conscious experience based on 96,000 hours of human heart record synchronized to Schuman Resonances, Solar Winds and Galactic Cosmic Rays and the resonance of those cosmic frequencies with human HRV, and the potential role of those whispering subsonic frequencies in global interconnectedness, open new era of human perception of the universe. Our work as a ring in the golden chain established in 1920 by A L Chizhevsky, supported by the new discoveries in HRV arena with the evidence of direct mathematical relationship between the HRV patterns and the spectral information encoded in the magnetic field, containing our magnetic potentials of our emotions and consciousness pillars are the bases of our new theory in the field: The Heart Based Resonant 


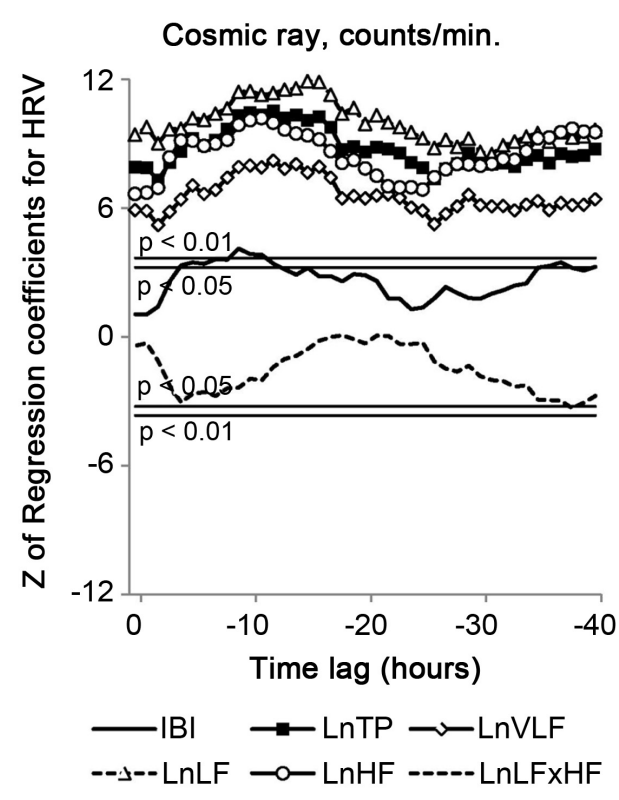

(a)

F10.7 cm solar radio flux

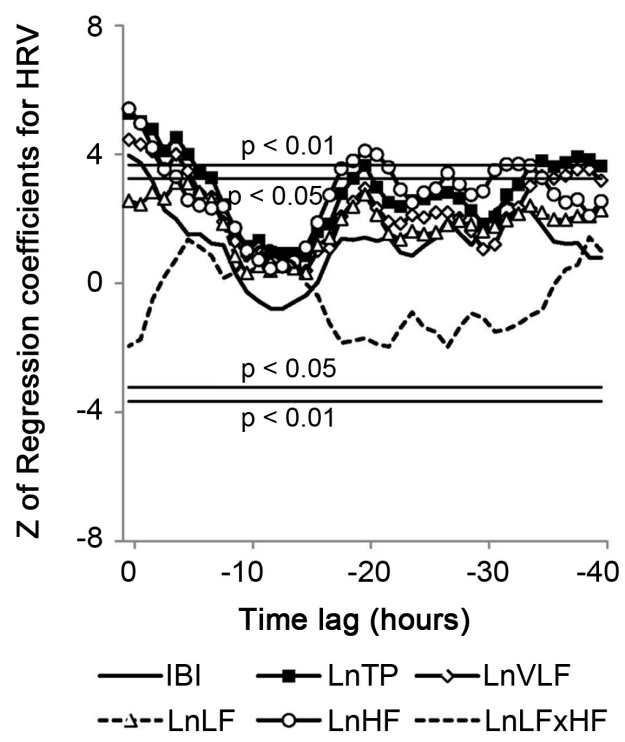

(c)

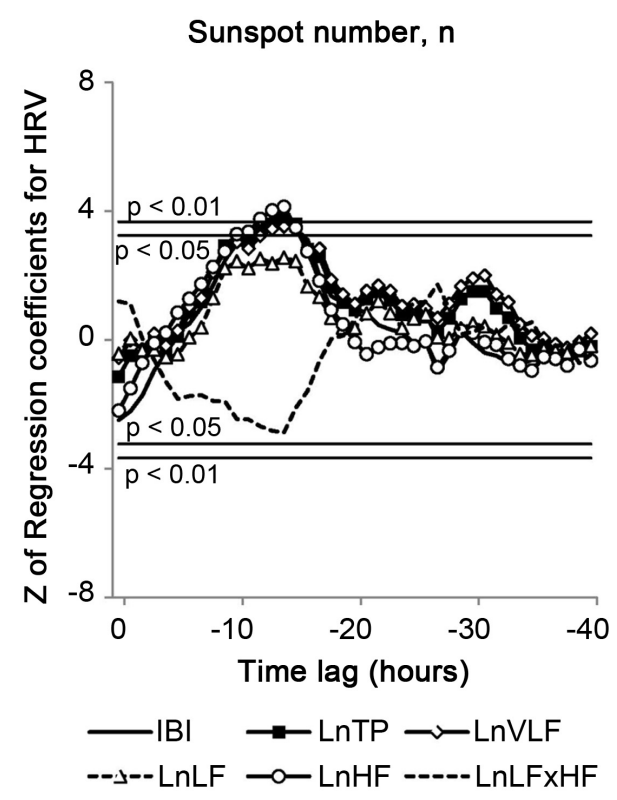

(b)

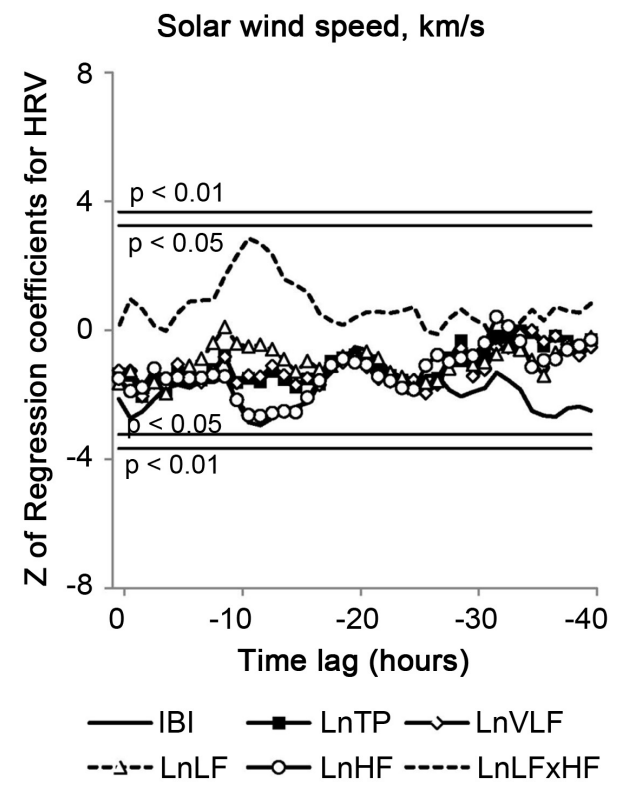

(d)

Figure 9. Our long term data record showing the correlations between HRV variables and changes in measures of solar activity and cosmic rays (HRV indices are: IBI: inter beat interval, TP: total power, LF: low frequency, HF: high frequency, VLF: very low frequency).

Frequencies Theory of consciousness (HBRF). Our theory of consciousness is renaissance of the ancient civilizations wisdom alerting to heart intelligence [43] and is complementary but not competitive to the models and theories of quantum consciousness by David Boehm, Karl Pribram, Penrose-Hamerof, Hiroomi Umezawa and others. The human heart with its dominant pulsating electromagnetic forces encoding human emotions and its delicate resonances in subsonic bands with Schumann Resonances, Solar Winds and to the extreme astronomic distant nuclear explosions of the endless galaxies represent tremendously organized universe with those sub sonic frequencies orchestrating what seems to be the great 
venerable whisper of the universal human consciousness.

\section{CONFLICTS OF INTEREST}

The authors declare no conflicts of interest regarding the publication of this paper.

\section{REFERENCES}

1. Al Abdulgader, A.A. (2020) Human Consciousness: The Role of Cerebral and Cerebellar Cortex, Vagal Afferents, and Beyond. In: Baloyannis, S., Ed., Cerebral and Cerebellar Cortex-Interaction and Dynamics in Health and Disease, IntechOpen, London. https://doi.org/10.5772/intechopen.95040

2. Alabdulgader, A.A. (2020) Human Consciousness: The Universal Heart Based Resonant Frequencies and the Massive Ecosystems Hierarchy. Archives in Neurology and Neuroscience, 9, 7 p. https://doi.org/10.33552/ANN.2020.09.000709

3. Alabdulgader, A.A. (2017) The Human Heart Rate Variability; Neurobiology of Psychophysiological Well Being and Planetary Resonance. General Internal Medicine and Clinical Innovations, 2, 2-4. https://doi.org/10.15761/GIMCI.1000141

4. Alabdulgader, A. (2012) Coherence: A Novel Nonpharmacological Modality for Lowering Blood Pressure in Hypertensive Patients. Global Advances in Health and Medicine, 1, 54-62.

https://doi.org/10.7453/gahmj.2012.1.2.011

5. Alabdulgader, A. (2016) Modulation of Heart Rate Variability: A Novel Non Pharmacological Modality for Lowering Blood Pressure in Hypertensive Patients. 9th Annual Meeting on Arrhythmia and Cardiac Surgery, Brisbane, 14-15 July 2016, 35.

6. Alabdulgader, A., McCraty, R., Atkinson, M., Dobyns, Y., Vainoras, A., Ragulskis, M., et al. (2018) Long-Term Study of Heart Rate Variability Responses to Changes in the Solar and Geomagnetic Environment. Scientific Reports, 8, Article No. 2663. https://www.nature.com/articles/s41598-018-20932-x https://doi.org/10.1038/s41598-018-20932-x

7. McCraty, R., Atkinson, M., Tomasino, D. and Bradley, R. (2009) The Coherent Heart: Heart Brain Interactions, Psychophysiological Coherence, and the Emergence of System-Wide Order. Integral Review, 5, 10-115.

8. McCraty, R. (2004) The Energetic Heart: Bioelectromagnetic Communication within and between People. In: Rosch, P.J. and Markov, M.S., Eds., Bioelectromagnetic Medicine, Marcel Dekker, New York, 541-562.

9. Ogawa, T. and Tanaka, Y. (1970) Q Factors of the Schumann Resonances and Solar Activity. Special Contributions, Geophysical Institute, Kyoto University, Kyoto, No. 10, 21-28.

10. Sekiguchi, M., Hayakawa, M., Nickolaenko, A.P. and Hobara, Y. (2006) Evidence on a Link between the Intensity of Schumann Resonance and Global Surface Temperature. Annals of Geophysics, 24, 1809-1817. https://doi.org/10.5194/angeo-24-1809-2006

11. (1996) Heart Rate Variability: Standards of Measurement, Physiological Interpretation, and Clinical Use. Task Force of the European Society of Cardiology and the North American Society of Pacing and Electrophysiology. Circulation, 93, 1043-1065.

12. Berntson, G.G., Bigger, J.T., Eckberg, D.L., et al. (1997) Heart Rate Variability: Origins, Methods, and Interpretive Caveats. Psychophysiology, 34, 623-648. https://doi.org/10.1111/j.1469-8986.1997.tb02140.x

13. McCraty, R. (2016) Science of the Heart Exploring the Role of the Heart in Human Performance. Volume 2, HeartMath Institute, Boulder Creek.

14. Cherry, N. (2002) Schumann Resonances, a Plausible Biophysical Mechanism for the Human Health Effects of Solar/Geomagnetic Activity. Natural Hazards, 26, 279-331. https://doi.org/10.1023/A:1015637127504 
15. McCraty, R. and Shaffer, F. (2015) Heart Rate Variability: New Perspectives on Physiological Mechanisms, Assessment of Self-Regulatory Capacity, and Health Risk. Global Advances in Health and Medicine, 4, 46-61. https://doi.org/10.7453/gahmj.2014.073

16. Bradley, R.T., McCraty, R., Atkinson, M. and Tomasino, D. (2010) Emotion Self-Regulation, Psychophysiological Coherence, and Test Anxiety: Results from an Experiment Using Electrophysiological Measures. Applied Psychophysiology and Biofeedback, 35, 261-283. https://doi.org/10.1007/s10484-010-9134-x

17. McCraty, R. and Shaffer, F. (2015) Heart Rate Variability: New Perspectives on Physiological Mechanisms, Assessment of Self-Regulatory Capacity, and Health Risk. Global Advances in Health and Medicine, 4, 46-61. https://doi.org/10.7453/gahmj.2014.073

18. Timofejeva, I., McCraty, R., Atkinson, M., Joffe, R., Vainoras, A., Alabdulgader, A.A. and Ragulskis, M. (2017) Identification of a Group's Physiological Synchronization with Earth's Magnetic Field. International Journal of Environmental Research and Public Health, 14, 998. https://doi.org/10.3390/ijerph14090998

19. McCraty, R., Atkinson, M., Stolc, V., Alabdulgader, A.A., Vainoras, A. and Ragulskis, M. (2017) Synchronization of Human Autonomic Nervous System Rhythms with Geomagnetic Activity in Human Subjects. International Journal of Environmental Research and Public Health, 14, 770. https://doi.org/10.3390/ijerph14070770

20. Alabdulgade, A., McCraty, R., Atkinson, M., Vainoras, A., Berškiene, K., et al. (2015) Human Heart Rhythm Sensitivity to Earth Local Magnetic Field Fluctuations. Journal of Vibroengineering, 17, 3271-3278.

21. Halberg, F., Cornélissen, G., McCraty, R., Czaplicki, J. and Al-Abdulgader, A.A. (2011) Time Structures (Chronomes) of the Blood Circulation, Populations' Health, Human Affairs and Space Weather. World Heart Journal, 3, 73-114.

22. Al-Abdulgader, A.A., Guillaume, G.C. and Halberg, F. (2011) Vascular Variability Disorders in the Middle East: Case Reports. World Heart Journal, 4, 261-277.

23. Timofejeva, I., McCraty, R., Atkinson, M., Joffe, R., Alabdulgader, A.A., Vainoras, A., Landauskas, M. and Ragulskis, M. (2021) Global Study of Human Heart Rhythm Synchronization with the Earth's Time Varying Magnetic Field. Applied Sciences, 11, 2935. https://doi.org/10.3390/app11072935

24. Zenchenkoa, T.A., Medvedevaa, A., Khorsevab, N.I. and Breusb, T.K. (2014) Synchronization of Human Heart-Rate Indicators and Geomagnetic Field Variations in the Frequency Range of 0.5-3.0 mHz. Atmospheric and Oceanic Physics, 50, 736-744. https://doi.org/10.1134/S0001433814040094

25. Kolb, B. and Teskey, G.C. (2012) Age, Experience, Injury, and the Changing Brain. Developmental Psychobiology, 54, 311-325. https://doi.org/10.1002/dev.20515

26. Nitsch, R. and Stahnisch, F.W. (2018) Neuronal Mechanisms Recording the Stream of Consciousness-A Reappraisal of Wilder Penfield's (1891-1976) Concept of Experiential Phenomena Elicited by Electrical Stimulation of the Human Cortex. Cerebral Cortex, 28, 3347-3355. https://doi.org/10.1093/cercor/bhy085

27. Lacey, J.I. (1959) Psychophysiological Approaches to the Evaluation of Psychotherapeutic Process and Outcome. In: Rubinstein, E. and Parloff, M., Eds., Research in Psychotherapy, American Psychological Association, Washington, DC, 160-208. https://doi.org/10.1037/10036-010

28. Lacey, J.I., Kagan, J., Lacey, B.C. and Moss, H.A. (1963) The Visceral Level: Situational Determinants and Behavioral Correlates of Autonomic Response Patterns. In: Knapp, P.H., Ed., Expression of the Emotions in Man, International Universities Press, New York, 161-196.

29. Wolk, C. and Velden, M. (1989) Revision of the Baroreceptor Hypothesis on the Basis of the New Cardiac Cycle Effect. In: Psychobiology: Issues and Applications, Elsevier Science Publishers B.V., Amsterdam, 371-379.

30. Lane, R.D., Reiman, E.M., Ahem, G.L. and Thayer, J.F. (2001) Activity in Medial Prefrontal Cortex Correlates with Vagal Component of Heart Rate Variability during Emotion. Brain and Cognition, 47, 97-100. 
31. Kosel, M. and Schlaepfer, T.E. (2003) Beyond the Treatment of Epilepsy: New Applications of Vagus Nerve Stimulation in Psychiatry. CNS Spectrums, 8, 515-521. https://doi.org/10.1017/S1092852900018988

32. Groves, D.A. and Brown, V.J. (2005) Vagal Nerve Stimulation: Are View of Its Applications and Potential Mechanisms That Mediate Its Clinical Effects. Neuroscience \& Biobehavioral Reviews, 29, 493-500. https://doi.org/10.1016/j.neubiorev.2005.01.004

33. Tiller, W.A., McCraty, R. and Atkinson, M. (1996) Cardiac Coherence: A New, Noninvasive Measure of Autonomic Nervous System Order. Alternative Therapies in Health and Medicine, 2, 52-65.

34. Tchijevsky, A.L. (1971) Physical Factors of the Historical Process. Cycles, 22, 11-27. (de Smitt, V.P. Translation)

35. McCraty, R. and Deyhl, N. (2016) The Science of Interconnectivity Exploring the Human-Earth Connection. HeartMath Institute, Boulder Creek.

36. Lean, J. (2000) Evolution of the Sun's Spectral Irradiance since the Maunder Minimum. Geophysical Research Letters, 27, 2425-2428. https://doi.org/10.1029/2000GL000043

37. Tapping, K. (1987) Recent Solar Radio Astronomy at Centimeter Wavelengths: The Temporal Variability of the 10.7 cm Flux. Journal of Geophysical Research: Atmospheres, 92, 829-838. https://doi.org/10.1029/JD092iD01p00829

38. Blanchard, J.P. and Blackman, C.F. (1994) Clarification and Application of an Ion Parametric Resonance Model for Magnetic Field Interactions with Biological Systems. Bioelectromagnetics, 15, 217-38. Erratum in: Bioelectromagnetics, 15, 493. https://doi.org/10.1002/bem.2250150306

39. Khabarova, O. (2004) Investigation of the Tchizhevsky-Velhover Effect. Biophysics, 49, S60-S67.

40. Palmer, S.J., Rycroft, M.J. and Cermack, M. (2006) Solar and Geomagnetic Activity, Extremely Low Frequency Magnetic and Electric Fields and Human Health at the Earth's Surface. Surveys in Geophysics, 27, 557-595. https://doi.org/10.1007/s10712-006-9010-7

41. Nelson, R.D. (2020) The Global Consciousness Project's Event-Related Responses Look like Brain EEG Event-Related Potentials. Journal of Scientific Exploration, 34, 246-267. https://doi.org/10.31275/20201475

42. Brizhik, L., Del Giudice, E., Jørgensen, S.E., Marchettini, N. and Tiezzi, E. (2009) The Role of Electromagnetic Potentials in the Evolutionary Dynamics of Ecosystems. Ecological Modelling, 220, 1865-1869. https://doi.org/10.1016/j.ecolmodel.2009.04.017

43. Alabdulgader, A. (2021) The Ancient Wisdom at Intersection with Modern Cardiac Sciences. Cardiology \& Vascular Research, 5, 1-13. 